\title{
Effects of platelet-rich fibrin on healing of intra-bony defects treated with anorganic bovine bone mineral
}

\author{
Yasemin SEZGIN(a) \\ Ahu URAZ(b) \\ I. Levent TANER ${ }^{(b)}$ \\ Rana ÇULHAOĞLU(b)
}

(a)Başkent University, Faculty of Dentistry, Department of Periodontology, Ankara, Turkey.

(b)Gazi University, Faculty of Dentistry, Department of Periodontology, Ankara, Turkey.

Declaration of Interests: The authors certify that they have no commercial or associative interest that represents a conflict of interest in connection with the manuscript.

\section{Corresponding Author:}

Yasemin Sezgin

E-mail: yasemin_tocak@hotmail.com

htrp://doi.org/10.1590/1807-3107BOR-2017.vol31.0015

Submitted: Aug 25, 2016

Accepted for publication: Nov 21, 2016

Last revision: Dec 08, 2016

\begin{abstract}
Anorganic bovine bone mineral (ABBM) is extensively used in the treatment of intra-bony defects. Platelet-rich fibrin (PRF) is a new-generation platelet concentrate with a simplified technique. Although certain studies have reported the use of PRF in the treatment of intra-bony defects, to date, none of them have evaluated its additive effects with ABBM. Therefore, a randomised, split-mouth clinical trial was conducted to compare healing of intra-bony defects treated with an ABBM-PRF combination with healing of those treated with ABBM alone. By using a split-mouth design, 15 paired intra-bony defects were randomly treated with either ABBM alone (control group) or ABBM-PRF combination (test group). Following clinical parameters and radiographical measurements were recorded at baseline and 6 months after treatment: plaque index (PI), gingival index (GI), probing depth (PD), gingival recession (GR), clinical attachment level (CAL), vertical bone loss, depth of defect and defect angle. Preoperative clinical and radiographical measurements were similar for the test and control groups. Statistically significant reductions in GI, PD, CAL, vertical bone loss, depth of intra-bony defect and widening of defect angle were detected after treatment in both groups. With respect to inter-group analysis, gain in CAL was significantly greater in the test group than in the control group, whereas no inter-group differences were observed in any other parameter. The results of this study indicate that both therapies are effective in the treatment of intra-bony defects.
\end{abstract}

Keywords: Periodontics; Alveolar Bone Loss; Bone Regeneration; Fibrin.

\section{Introduction}

The risk of further alveolar bone loss and the probability of tooth loss increases with the presence of intra-bony defects. Hence, treatment of intra-bony defects comprises several treatment modalities aimed to reconstruct the attachment apparatus which includes bone grafts, guided tissue regeneration, biomolecular techniques, or combination of some of these approaches. ${ }^{1}$

Over the years, various types of graft materials, including autogenic bone, demineralised allogenic bone, xenogeneic materials and alloplastic materials, have been used to regenerate the attachment apparatus. Among xenogeneic materials, anorganic bovine bone mineral (ABBM) 
is a widely researched and well-documented grafting material. ${ }^{1} \mathrm{ABBM}$ is produced by removal of all organic components of cancellous or cortical bovine bone. It can be safely used as no systemic or local immune response was observed after ABBM implantation. ${ }^{2}$ Histologically, new bone, cementum and new periodontal ligament formation have been observed in periodontal defects treated with ABBM.,4 Considering its aforementioned properties, ABBM has been successfully used in regenerative treatment of intra-bony and furcation defects. ${ }^{1,5}$

In recent years, researchers have focused on biological mediators which have the ability to enhance wound healing and improve clinical benefits of bone replacement grafts. ${ }^{6}$ Polypeptide growth factors are biological mediators which regulate cell proliferation, chemotaxis and differentiation and induce periodontal regeneration. ${ }^{7}$

To deliver high concentrations of polypeptide growth factors to periodontal surgical wounds, use of autologous platelet concentrates serve as a safe and appropriate approach. Various platelet concentrates have been developed to improve soft and hard tissue healing. ${ }^{8}$ Platelet-rich fibrin (PRF) is a second-generation platelet concentrate, defined as autologous leukocyte- and platelet-rich fibrin because it collects leukocytes and platelets with high efficiency. PRF has been demonstrated to release polypeptide growth factors such as transforming growth factor $\beta 1$, platelet-derived growth factor, vascular endothelial growth factor and matrix glycoproteins (such as thrombospondin-1) gradually over at least 1 week. ${ }^{8,9}$

PRF has been used in different surgical procedures such as sinus floor augmentation, treatment of gingival recession (GR), furcation defects, maxillary rehabilitation before implant placement, restoration of peri-implant defects, as well as healing of extraction sockets with promising results. ${ }^{10,11,12,13,14}$ In addition, certain clinical studies have been conducted on the use of PRF for treatment of intra-bony defects in humans, $15,16,17,18,19$ but none have investigated its additive effects with ABBM for treatment of intra-bony defects. Thus, the present split-mouth, single-centre, controlled study aimed to compare the healing of intra-bony defects treated with a combination of ABBM-PRF or ABBM alone; the hypothesis being tested was that PRF would augment the regenerative effects of ABBM in human intra-bony defects.

\section{Methodology}

\section{Patient selection and experimental design}

Participants were recruited form patients referred to the Department of Periodontology, Gazi University, Turkey, and were enrolled after receiving detailed description of the procedures and providing signed informed consent. The study protocol was approved by the ethics board at the Faculty of Dentistry, Gazi University (2010/78). This parallel-arm clinical trial was registered at ClinicalTrials.gov (NCT02836314) and was conducted in accordance with the Helsinki Declaration of 1975, as revised in 2000. As far as possible, the CONSORT 2010 guidelines for reporting parallel-group randomised trials were followed. ${ }^{20}$ All patients complained about generalised, severe, chronic periodontal disease, classified as generalised when $>30 \%$ of sites were affected by a clinical attachment level (CAL) loss of $\geq 5 \mathrm{~mm} \cdot{ }^{21}$ The inclusion criteria of the study were as follows: no systemic diseases; a good level of oral hygiene $(\mathrm{OH})$ (plaque index $[\mathrm{PI}]<0.15)$; presence of two paired, two- or three-wall intra-bony defects with a probing depth (PD) of $\geq 6 \mathrm{~mm}$ and an intra-bony component of $\geq 3 \mathrm{~mm}$, as detected on radiographs; no intra-bony defects extending into the furcation area; tooth mobility $\leq 1$; tooth and adjoining teeth testing vital and without symptoms or signs of endodontic involvement; and tooth and adjoining teeth free of caries or inadequate restorations.

Exclusion criteria included the following: patients with compromised immune systems; pregnant and/or lactating women; patients taking any drug known to affect the periodontal status or the coagulation system; and smokers.

This clinical study was a randomised controlled trial with a split-mouth design. The selected sites were randomly (coin toss) divided into control (ABBM alone) and test (ABBM-PRF) groups.

\section{Pre-surgical therapy}

Before surgery, each patient received cause-related periodontal treatment consisting of $\mathrm{OH}$ instructions, 
motivation, full-mouth supra- and sub-gingival scaling and root planning. In cases diagnosed with trauma from occlusion, occlusal adjustment was performed. Four weeks after phase I periodontal therapy (baseline), periodontal re-evaluation was performed to confirm suitability of the sites for the surgical study.

\section{Intra-examiner reproducibility}

Two periodontists performed all surgeries. One examiner other than the surgeons performed all clinical measurements, and another examiner performed all radiographical measurements. Both examiners were blinded to the study groups. Before surgery, both examiners evaluated pre-surgical measurements from five patients and five digital radiographs twice, 48 hours apart. Calibration was accepted if $\geq 90$ of the recordings could be reproduced within a 1-mm difference.

\section{Clinical measurements}

Following clinical parameters were assessed at baseline and 6 months after surgery by using a periodontal probe (Nordent Manufacturing Inc., Illinois, USA) by a calibrated investigator other than the surgeons who performed the surgeries: PI, gingival index (GI), PD, CAL and GR. ${ }^{22-23}$ All clinical measurements were recorded with the aid of an acrylic stent, for which, occlusal acrylic stents were fabricated and grooves were placed to standardise the position of the periodontal probe.

\section{Radiographical measurements}

Standardised radiographical examination was simultaneously conducted with clinical measurements. Radiographs were taken using long-cone parallel and direct digital radiography. An x-ray-sensitive intra-oral digital radiography sensor (RVG 5000, Eastman Kodak, Rochester, NY, U.S.A), an optimal standardisation and paralleling system (XCP-DS ${ }^{\circledast}$, Sendor Positioning System for Kodak Trophy $\mathrm{RVG}^{\circledR}$ Digital Sensors, Dentsply Rinn ${ }^{\circledR}$ Dentsply Int, Inc.) and digital imaging software (Sigma Scan Pro 5 Diagnostic Systems, Dept. Oral Radiology/ACTA, Amsterdam, Holland) were used. Site-specific individual bite stents were prepared from silicone elastomeric material (Zetaplus, Zhermack, Rovigo, Italy) for optimal localisation of the sensor positioning system in each defect area. The following parameters were assessed:

a. Vertical bone loss: distance between cemento-enamel junction and base of the defect (CEJ-BD) (Figure 1); ${ }^{24}$

b. Depth of intra-bony defect (DD): distance between alveolar crest and base of the defect (AC-BD) (Figure 1); ${ }^{25}$

c. Radiographical defect angle: by using the function 'angle', the defect angle was measured by assessing the two lines which represent the root surface of the involved tooth and the bone defect surface (Figure 1). ${ }^{26}$

\section{Surgical therapy}

Following local anaesthesia, buccal and lingual sulcular incisions were made, and mucoperiosteal flaps were elevated. The flap was extended one or two teeth mesially or distally to achieve better access to the surgical site. Granulation tissue was removed from the defects, and the roots were scaled and planed using hand instruments; however, the root surfaces were not conditioned.

In the test group, blood samples were obtained according to the PRF protocol which was developed by Choukroun et al. and immediately centrifuged at 2,700 rpm for 12 minutes (approximately $400 \mathrm{~g}$ ) by PC-02 table centrifuge, specifically designed for this application (Process, Nice, France). ${ }^{27}$ PRF clots were easily separated from red corpuscles base by using sterile tweezers and scissors and then transformed into fibrin membranes by using the PRF Box (Process, Nice, France), designed to collect and standardise PRF clots and membranes in order to avoid extracting and losing a significant amount of growth factors. ${ }^{28}$ One PRF was cut by scissors to produce small pieces and then mixed with ABBM granules with a particle size of $0.25-1 \mathrm{~mm}$ (Bio-Oss, Gesitlich, Switzerland) in a sterile dappen dish; this mixture was added to the defect by means of a sterile curette. The defect was filled to the existing alveolar crest. The other PRF was used to cover the defect as a barrier. 


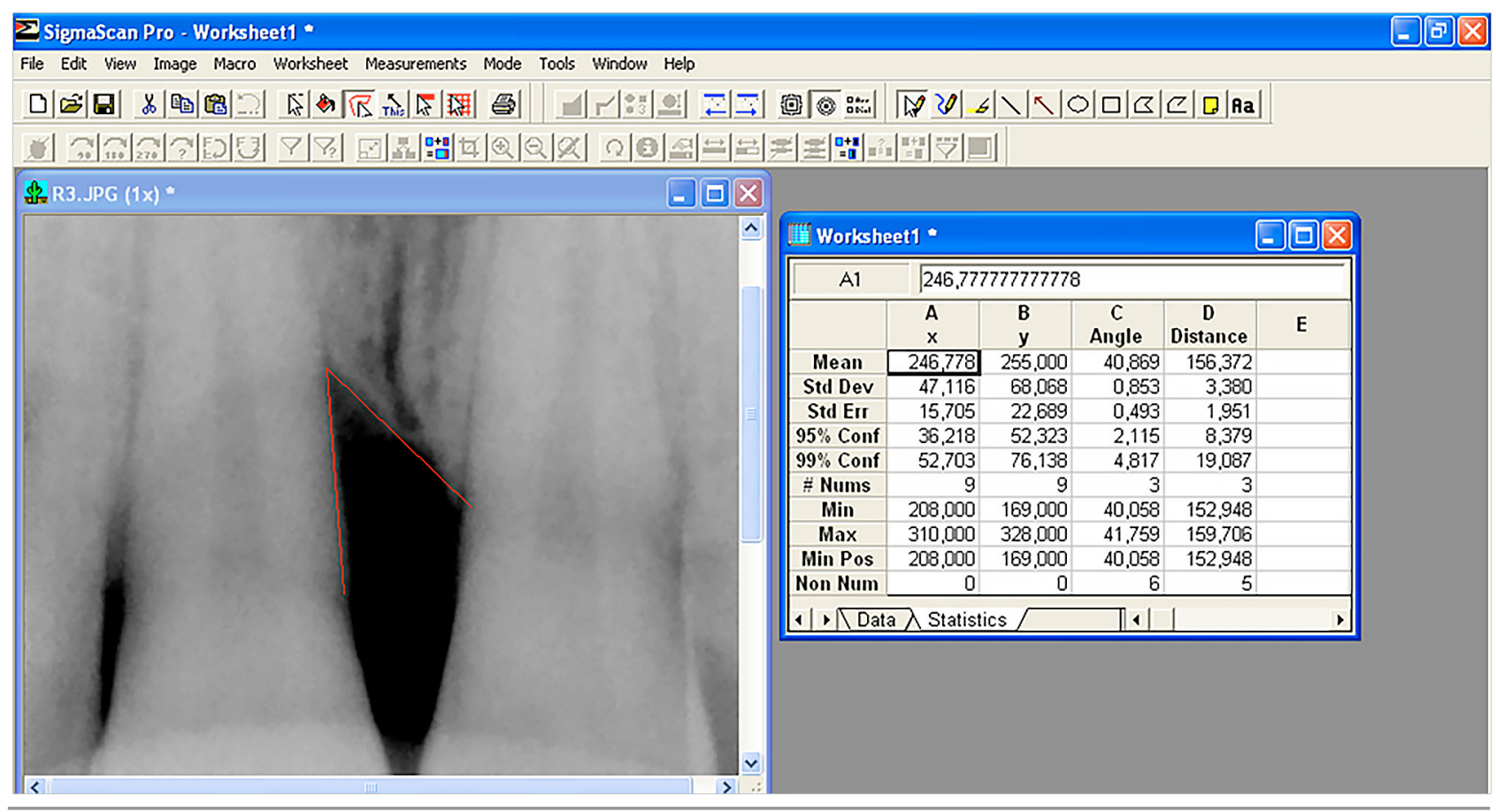

Figure 1. Measurements of vertical bone loss and radiographical defect angle with digital radiography imaging software.

In the control group, ABBM was prepared by pouring the particles into a sterile dappen dish and rehydrated with sterile saline, and graft material was placed on the defect; the defect was filled to the existing alveolar crest.

Primary soft tissue closure was obtained using 4-0 silk suture, and periodontal dressing was placed over the surgical area to achieve haemostasis and wound stability in both groups.

\section{Post-surgical care}

Patients were instructed to avoid brushing and flossing at the surgical site until the sutures and periodontal dressing were removed. All patients were prescribed systemic antibiotics (doxycycline: $200 \mathrm{mg}$ on the day of surgery and $100 \mathrm{mg} /$ day thereafter for 14 days) and $0.2 \%$ chlorhexidine gluconate rinses (every 12 hours for 4 weeks). Patients were also prescribed oral analgesics (paracetamol $100 \mathrm{mg}$, as needed). The periodontal dressing and sutures were removed 1 week after surgery. Surgical areas were cleansed with $0.12 \%$ chlorhexidine digluconate, and patients were advised gentle brushing with a soft toothbrush.
Patients were examined weekly up to 1 month after surgery and then at 2, 3 and 6 months.

\section{Statistical analysis}

Descriptive statistics were expressed as mean \pm standard deviation (SD). Statistical analysis was performed using a software program (Minitab version 16.1.1, USA). The normality of values was tested using the Anderson-Darling test. PD, CAL, GR, vertical bone loss, depth of the intra-bony defect and radiographical defect angle values were normally distributed and compared using paired samples t-test within each group and between groups at baseline and at 6 months. GI and PI variables were not normally distributed, and the Wilcoxon test was used to detect significant differences within and between groups at baseline and at 6 months. $p$ values $<0.05$ were considered statistically significant.

\section{Sample size calculation}

Sample size calculation suggested that a minimum of 14 patients were needed to demonstrate a $1 \mathrm{~mm}$ difference in CAL between the two groups after treatment (90\% power, $\alpha$ of $0.05, \mathrm{SD}$ of 1.06$).{ }^{17}$ 
Considering the dropout rate, to achieve at least 14 patients, a total sample of 21 patients were enrolled in this study.

\section{Results}

Of the 30 screened patients, 21 eligible participants who provided consent were randomised into test $(n=21)$ or control $(n=21)$ groups and 15 patients completed the study ( 7 women and 8 men; age: 38-61 years). Five patients were excluded on account of defects which did not meet the study criteria at the time of surgery, and one patient did not return for the follow-up examination (Figure2). All sites healed uneventfully with no clinically detectable or subjectively reported side effects.

The defects displayed comparable distribution in the two groups (Table 1). Initial clinical and radiographical measurements were similar in both test and control groups, and no statistical difference was observed between the groups $(p>0.05)$ (Table 2).

The difference in PI scores at baseline and 6 months after treatment was not significant in
Table 1. Distribution of treated defects $(n=15)$.

\begin{tabular}{lcc}
\hline Defect Location & Test group & Control group \\
\hline Maxilla & 8 & 6 \\
Mandible & 7 & 9 \\
Anterior teeth & 6 & 6 \\
Premolars & 7 & 6 \\
Molars & 2 & 3 \\
\hline
\end{tabular}

Table 2. Baseline clinical and radiographical measurements.

\begin{tabular}{lcc}
\hline Parameter & Test group & Control group \\
\hline $\mathrm{PI}$ & $0.07 \pm 0.25$ & $0.13 \pm 0.35$ \\
$\mathrm{GI}$ & $0.93 \pm 0.59$ & $0.94 \pm 0.01$ \\
$\mathrm{PD}(\mathrm{mm})$ & $7.46 \pm 1.19$ & $7.07 \pm 1.03$ \\
$\mathrm{GR}(\mathrm{mm})$ & $0.87 \pm 0.91$ & $0.66 \pm 0.72$ \\
$\mathrm{CAL}(\mathrm{mm})$ & $8.33 \pm 1.76$ & $7.73 \pm 1.16$ \\
Vertical bone loss $(\mathrm{mm})$ & $8.44 \pm 2.13$ & $8 \pm 1.71$ \\
Depth of the defect $(\mathrm{mm})$ & $4.33 \pm 0.93$ & $3.64 \pm 0.93$ \\
Defect angle $\left(^{\circ}\right)$ & $37.87 \pm 5.25$ & $41.37 \pm 5.92$ \\
\hline
\end{tabular}

Values are presented as mean \pm standard deviation; PI: plaque index; GI: gingival index; PD: pocket depth; GR: gingival recession; CAL: clinical attachment level.

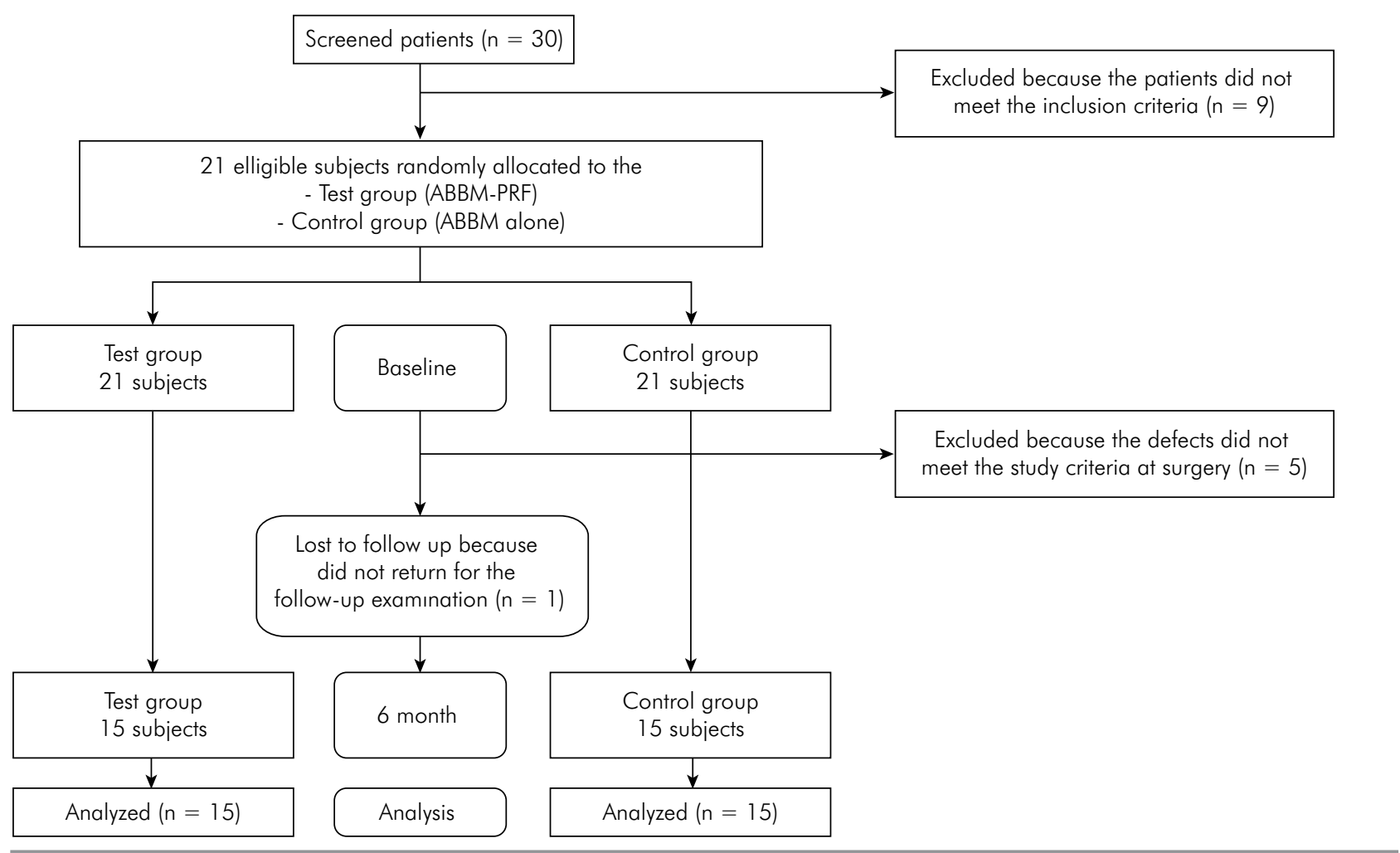

Figure 2. Consort flow chart of the study. 
any group, whereas this difference in GI scores was significant in both groups $(p<0.05$; Table 3$)$. Moreover, PD decreased significantly compared to baseline at 6 months after treatment in both groups $(p<0.05)$, but the inter-group differences were not statistically significant at any evaluation time point ( $\mathrm{p}>0.05$; Table 4).

In terms of CAL gain, both groups demonstrated significant improvements compared to baseline. In inter-group analysis, significantly greater CAL gain was detected in the test group than in the control group ( $\mathrm{p}<0.05$; Table 4$)$.
In addition, increase in GR was not statistically significant at 6 months after treatment compared to baseline ( $p>0.05)$ in the test group, whereas this increase was statistically significant in the control group $(\mathrm{p}<0.05)$; however, the inter-group difference in these increases was not statistically significant at any evaluation time point ( $\mathrm{p}>0.05$; Table 4 ).

According to hard tissue findings, both treatment groups showed significant improvements compared to baseline in terms of vertical bone gain, defect fill and defect angle at 6 months after treatment $(\mathrm{p}<0.05$; Figures 3 and 4).

Table 3. Plaque index and gingival index scores in both groups.

\begin{tabular}{lcccc}
\hline Parameter & Baseline test group & 6 Months test group & Baseline control group & 6 Months control group \\
$\mathrm{PI}$ & $0.07 \pm 0.25$ & $0.13 \pm 0.35$ & $0.13 \pm 0.35$ & $0.13 \pm 0.35$ \\
$\mathrm{GI}$ & $0.93 \pm 0.59$ & $0.27 \pm 0.46^{*}$ & $0.94 \pm 0.01$ & $0.28 \pm 0.01^{*}$ \\
\hline
\end{tabular}

PI: plaque index, GI: gingival index; *statistically significant difference compared to baseline $(p<0.05)$.

Table 4. Mean and standard deviation of clinical and radiographical measurements in both groups.

\begin{tabular}{|c|c|c|c|c|}
\hline Parameter & Baseline & 6 months & Difference & $p$-value \\
\hline \multicolumn{5}{|l|}{$\mathrm{PD}(\mathrm{mm})$} \\
\hline Test group & $7.46 \pm 1.19$ & $2.53 \pm 0.74^{*}$ & $4.93 \pm 1.22$ & $<0.001$ \\
\hline Control group & $7.07 \pm 1.03$ & $2.86 \pm 1.12^{*}$ & $4.21 \pm 1.21$ & $<0.001$ \\
\hline$p$-value & NS & NS & NS & \\
\hline \multicolumn{5}{|l|}{$\mathrm{GR}(\mathrm{mm})$} \\
\hline Test group & $0.87 \pm 0.91$ & $1.33 \pm 0.82$ & $0.46 \pm 0.83$ & NS \\
\hline Control group & $0.66 \pm 0.72$ & $1.60 \pm 0.63^{*}$ & $0.94 \pm 0.70$ & $<0.001$ \\
\hline $\mathrm{p}$-value & NS & NS & NS & - \\
\hline \multicolumn{5}{|l|}{$\mathrm{CAL}(\mathrm{mm})$} \\
\hline Test group & $8.33 \pm 1.76$ & $3.86 \pm 1.19^{*}$ & $4.47 \pm 1.60^{\circ}$ & $<0.001$ \\
\hline Control group & $7.73 \pm 1.16$ & $4.46 \pm 1.35^{*}$ & $3.27 \pm 1.34^{\pi}$ & $<0.001$ \\
\hline$p$-value & NS & NS & $<0.05$ & - \\
\hline \multicolumn{5}{|c|}{ Vertical Bone Loss (mm) } \\
\hline Test group & $8.44 \pm 2.13$ & $4.08 \pm 1.09^{*}$ & $4.36 \pm 2.42$ & $<0.001$ \\
\hline Control group & $8 \pm 1.71$ & $4.06 \pm 0.91^{*}$ & $3.94 \pm 1.69$ & $<0.001$ \\
\hline$p$-value & NS & NS & NS & - \\
\hline \multicolumn{5}{|c|}{ Depth of the Defect $(\mathrm{mm})$} \\
\hline Test group & $4.33 \pm 0.93$ & $1.78 \pm 0.39^{*}$ & $2.55 \pm 1.15$ & $<0.001$ \\
\hline Control group & $3.64 \pm 0.93$ & $1.66 \pm 0.42^{*}$ & $1.98 \pm 0.80$ & $<0.001$ \\
\hline$p$-value & NS & NS & NS & - \\
\hline \multicolumn{5}{|l|}{ Defect Angle $\left({ }^{\circ}\right)$} \\
\hline Test group & $37.87 \pm 5.25$ & $51.15 \pm 4.02^{*}$ & $13.28 \pm 8.27$ & $<0.001$ \\
\hline Control group & $41.37 \pm 5.92$ & $53.09 \pm 4.03^{*}$ & $11.72 \pm 6.83$ & $<0.001$ \\
\hline $\mathrm{p}$-value & NS & NS & NS & - \\
\hline
\end{tabular}

*statistically significant difference between the groups $(p<0.05)$ 


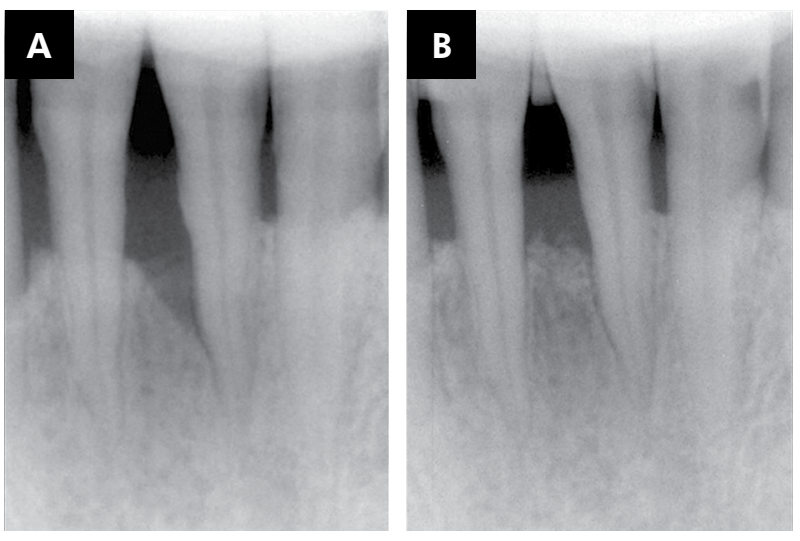

Figure 3. Test group: (A) periapical radiograph of the intra-bony defect at baseline; $(B)$ periapical radiograph of the intra-bony defect at 6 months after ABBM-PRF combination treatment.

\section{Discussion}

The present randomised clinical trial compared the use of ABBM alone (control group) to ABBM-PRF combination (test group) in surgical treatment of intra-bony defects. Statistically significant reductions in GI, PD, CAL, vertical bone loss, depth of the intra-bony defect and widening of the defect angle were detected after treatment in both groups. GR increased significantly in the control group, whereas this increase was not significant in the test group. According to inter-group analysis, only CAL gain was statistically significant in the test group compared to the control group, whereas other parameters did not show significant inter-group differences.

To the best of our knowledge, to date, there have been no published reports which compare clinical and radiographical effects of $A B B M$ with those of ABBM-PRF in the treatment of periodontal intra-bony defects. Thus, a direct comparison with other studies was not possible.

At baseline, none of the measured clinical and radiographical variables showed any significant inter-group differences, thus ensuring the same starting point for both procedures and the prevention of selection bias. ${ }^{29}$

In the present study, mean PD decreased from $7.46 \pm 1.19 \mathrm{~mm}$ to $2.53 \pm 0.74 \mathrm{~mm}$ at the test sites and from $7.07 \pm 1.03 \mathrm{~mm}$ to $2.86 \pm 1.12 \mathrm{~mm}$ at the control sites at 6 months after surgery; however, these
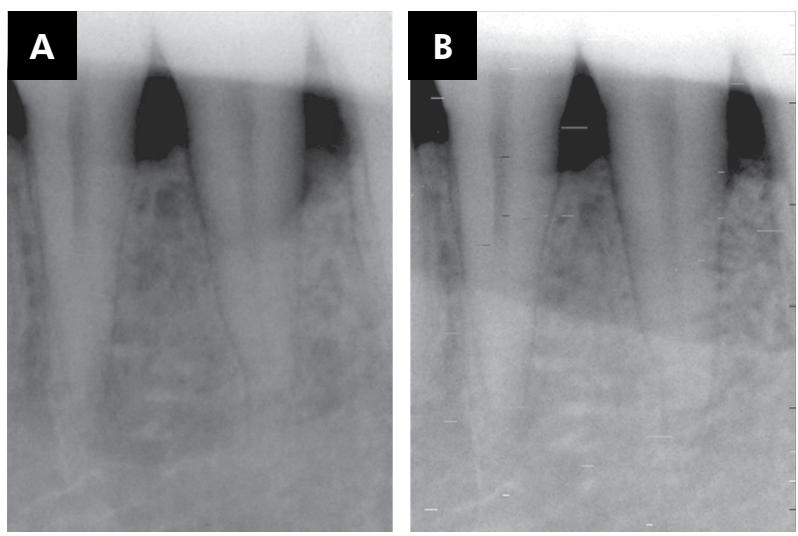

Figure 4. Control group: (A) periapical radiograph of the intra-bony defect at baseline; (B) periapical radiograph of the intra-bony defect at 6 months after ABBM treatment.

differences were not statistically significant between the two groups. Döri et al. ${ }^{30}$ compared ABBM with ABBM-PRP in human intra-bony defects and reported no significant differences between the two treatment groups. In the first year, PD reduction was $5.2 \mathrm{~mm}$ for the test group and $5.3 \mathrm{~mm}$ for the control group. In another controlled clinical comparative study ${ }^{31}$ conducted by Döri et al. ${ }^{30}$ the PD reduction was $6.20 \mathrm{~mm}$ for the control group and $6.60 \mathrm{~mm}$ for the test group at 9 months after surgery. These reductions were greater than those obtained in the present study. Conversely, Richardson et al. ${ }^{32}$ compared ABBM to demineralised, freeze-dried bone allograft (DFDBA) in human intra-bony defects and 6 months after surgery, the PD reduction was $3 \mathrm{~mm}$ for the ABBM group, which are lower compared to the reductions obtained in the present study. This discrepancy could be attributed to the differences in terms of study design, re-evaluation period, inclusion criteria, initial soft tissue measurements, initial defect depth and intra-bony defect configuration. ${ }^{33}$

In the control group, the increase in GR was statistically significant compared to baseline, whereas in the test group, this increase was not statistically significant compared to baseline; this could be attributed to placement of the PRF membrane over the alveolar crest in the test group. In this way, PRF acts as a healing and inter-position biomaterial owing to its mechanical adhesive properties and biological functions similar to fibrin glue. ${ }^{34}$ 
A greater and statistically significant CAL gain was noted in the test group $(4.47 \pm 1.60 \mathrm{~mm})$ compared to the control group $(3.27 \pm 1.34 \mathrm{~mm})$ at 6 months after surgery. As described earlier, by using PRF membrane in the test group, GR remained stable after surgery, whereas it increased significantly in the control group. The significant CAL gain observed in the test group could be explained by the achievement of lesser GR at 6 months after surgery owing to use of PRF membrane.

In terms of vertical bone gain, the results of this study compare favourably with those reported in previous human studies with ABBM. ${ }^{32,35}$ Conversely, the reduction in the depth of the defect obtained in the present study is lower compared to the reductions observed in a previous study which reported a 3.8-mm defect fill at the 18-month evaluation of defects treated with a DFDBA-PRP combination..$^{36}$ This can be attributed to the difference in the re-evaluation period, graft material and initial defect depth.

Klein et al. ${ }^{37}$ reported a defect angle gain of $12.81^{\circ}$ in intra-bony defects treated with GTR. Similarly, Ilgenli et al. ${ }^{36}$ reported a defect angle gain of $10.8^{\circ}$ at 18 months after surgery in intra-bony defects treated with a DFDBA-PRP combination. In the present study, the defect angle increased from $37.87 \pm 5.25^{\circ}$ to $51.15 \pm 4.02^{\circ}$ in the test group and from $41.37 \pm 5.92^{\circ}$ to $53.09 \pm 4.03^{\circ}$ in the control group. These results are in concordance with those of the aforementioned studies. ${ }^{36,37}$

Several studies ${ }^{15,16,17,38}$ have examined the suitability of autologous PRF for treatment of human intra-bony defects and revealed that PRF improves clinical ${ }^{15}$ and radiographical ${ }^{15,16,17,38}$ parameters. However, in the present study, no statistically significant differences were observed between both groups, except for the significant CAL gain noted in the test group. This discrepancy is likely because of the disparity of the study designs. In previous studies, the control groups were treated with conventional open-flap debridement alone or with PRF, whereas in the present study, the control group was treated with ABBM. ABBM is an extensively researched material which has been successfully used alone for treatment of intra-bony and furcation defects, with a positive role in reducing $\mathrm{PD}$, promoting CAL gain and enhancing defect fill in periodontal defects. $3,4,5,32$ The positive results of the control group (ABBM alone) are in accordance with findings from previous studies which had failed to show significant differences between treatment with ABBM alone or combinations of ABBM with enamel matrix derivative or platelet-rich plasma (PRP). ${ }^{30,39}$ Although the results of this study suggest that treatment with ABBM-PRF may not have provided significantly better results than those obtained with ABBM alone, the possibility for PRF to show any beneficial effects over the control group might have been masked by the fact that treatment with ABBM alone resulted in significant improvement.

The present study has several important strengths. It was designed as a split-mouth study to maintain a powerful estimate of treatment effect with a smaller sample size and discard individual patient characteristics. In addition, different examiners blinded to study groups performed all clinical and radiological measurements. Moreover, by using Choukroun's protocol to obtain PRF and by using PRF Box when transforming PRF clots to PRF membrane are some of the other strengths of this study. Choukroun's protocol does not require anticoagulants or bovine thrombin but requires a PC-02 table centrifuge and a collection kit from Process (Nice, France); thus, PRF has the characteristic of polymerising naturally and slowly during centrifugation. This type of polymerisation facilitates PRF to progressively release growth factors which play essential roles in cell proliferation, chemotaxis and differentiation and induce periodontal regeneration. ${ }^{7,89}$ In order to avoid extraction and losing a significant amount of growth factors, Choukroun invented the PRF Box; this standardised and gentle compression approach allows an increase in the release of total growth factors by the PRF membrane. ${ }^{28}$ Both obtaining and compression methods influence the total amount and releasing profile of growth factors in PRF. ${ }^{27,28}$ Therefore, following Choukroun's simple protocol principles is necessary to obtain reproducible and reliable results. However, our study also has certain limitations. The first limitation is the short evaluation period of 6 months. Moreover, lack of histological examination to evaluate the regenerative capacity of PRF and lack of evaluation of patient-reported outcomes may be rendered as study limitations.

In conclusion, results of this study suggest that both ABBM alone and ABBM combined with PRF can be 
successfully used for treatment of intra-bony defects. On comparing sites treated with ABBM alone versus ABBM-PRF combination, no statistically significant differences were found in clinical and radiographical parameters, except for statistically significant CAL gain in the test group. The similar results observed in both groups can be attributed to two possible reasons: first, the use of $\mathrm{ABBM}$ alone in the control group might have masked any positive effects of PRF in the treatment; the second reason might be lack of evaluation of patient-reported outcomes and soft tissue characteristics. Patient perceptions could have been quantified by administering a self-report questionnaire by using a visual analogue scale (VAS) which tests for variables of pain, sensitivity, swelling and discomfort experienced during the procedures, whereas soft tissue characteristics could have been evaluated using pink aesthetic score. By evaluating

\section{References}

1. Slotte C, Asklöw B, Sultan J, Norderyd O. A randomized study of open-flap surgery of 32 intrabony defects with and without adjunct bovine bone mineral treatment. J Periodontol. 2012;83(8):999-1007. http://doi.org/10.1902/jop.2011.110490

2. Cohen RE, Mullarky RH, Noble B, Comeau RL, Neiders ME. Phenotypic characterization of mononuclear cells following anorganic bovine bone implantation in rats. J Periodontol. 1994;65(11):1008-15. http://doi.org/10.1902/jop.1994.65.11.1008

3. Mellonig JT. Human histologic evaluation of a bovine-derived bone xenograft in the treatment of periodontal osseous defects. Int J Periodontics Restorative Dent. 2000;20(1):19-29.

4. Camelo M, Nevins ML, Schenk RK, Simion M, Rasperini G, Lynch SE et al. Clinical, radiographic, and histologic evaluation of human periodontal defects treated with Bio-Oss and Bio-Gide. Int J Periodontics Restorative Dent. 1998;18(4):321-31.

5. Lekovic V, Camargo PM, Weinlaender M, Vasilic N, Aleksic Z, Kenney EB. Effectiveness of a combination of platelet-rich plasma, bovine porous bone mineral and guided tissue regeneration in the treatment of mandibular grade II molar furcations in humans. J Clin Periodontol. 2003;30(8):746-51. http://doi.org/10.1034/j.1600-051X.2003.00368.x

6. Nevins ML, Reynolds MA. Tissue engineering with recombinant human platelet-derived growth factor $\mathrm{BB}$ for implant site development. Compend Contin Educ Dent. 2011;32(2):20-7. these parameters, significant differences could have been detected. Thus, additional controlled studies are warranted in the future to evaluate patient-centred outcomes and soft tissue characteristics in order to confirm these results with larger samples and longer evaluation periods.

\section{Conclusion}

The results of this study indicate that both ABBM and ABBM-PRF interventions are effective in the treatment of intra-bony defects. However, addition of PRF to ABBM may lead to enhancement of CAL gain.

\section{Acknowledgements}

This research was funded by Gazi University Research Grant (03/10-12). The authors would like to thank Çağın Sezgin for his invaluable contributions.
7. Santana RB, Santana CM. Human intrabony defect regeneration with rhFGF-2 and hyaluronic acid - a randomized controlled clinical trial. J Clin Periodontol. 2015;42(7):658-65. http://doi.org/10.1111/jcpe.12406 PMID:25933201

8. Dohan Ehrenfest DM, Bielecki T, Del Corso M, Inchingolo F, Sammartino G. Shedding light in the controversial terminology for platelet-rich products: platelet-rich plasma (PRP), platelet-rich fibrin (PRF), platelet-leukocyte gel (PLG), preparation rich in growth factors (PRGF), classification and commercialism. J Biomed Mater Res A. 2010;95(4):1280-2. http://doi.org/10.1002/jbm.a.32894

9. Dohan Ehrenfest DM, de Peppo GM, Doglioli P, Sammartino G. Slow release of growth factors and thrombospondin-1 in Choukroun's platelet-rich fibrin (PRF): a gold standard to achieve for all surgical platelet concentrates technologies. Growth Factors. 2009;27(1):63-9. http://doi.org/10.1080/08977190802636713

10. Moraschini V, Barboza ES. Use of platelet-rich fibrin membrane in the treatment of gingival recession: a systematic review and meta-analysis. J Periodontol. 2016;87(3):281-90. http://doi.org/10.1902/jop.2015.150420

11. Sharma A, Pradeep AR. Autologous platelet-rich fibrin in the treatment of mandibular degree II furcation defects: a randomized clinical trial. J Periodontol. 2011;82(10):1396-403. http://doi.org/10.1902/jop.2011.100731 
12. Simonpieri A, Del Corso M, Sammartino G, Dohan Ehrenfest DM. The relevance of Choukroun's platelet-rich fibrin and metronidazole during complex maxillary rehabilitations using bone allograft. Part I: a new grafting protocol. Implant Dent. 2009;18(2):102-11. http://doi.org/10.1097/ID.0b013e318198cf00

13. Jang ES, Park JW, Kweon H, Lee KG, Kang SW, Baek DH et al. Restoration of peri-implant defects in immediate implant installations by Choukroun platelet-rich fibrin and silk fibroin powder combination graft. Oral Surg Oral Med Oral Pathol Oral Radiol Endod. 2010;109(6):831-6. http://doi.org/10.1016/j.tripleo.2009.10.038

14. Kotsakis GA, Boufidou F, Hinrichs JE, Prasad HS, Rohrer M, Tosios KI. Extraction socket management utilizing platelet rich fibrin: a proof-of-principle study of the "Accelerated-early implant placement" concept. J Oral Implantol. 2016;42(2):164-8. http://doi.org/10.1563/aaid-joi-D-15-00001

15. Thorat M, Pradeep AR, Pallavi B. Clinical effect of autologous platelet-rich fibrin in the treatment of intra-bony defects: a controlled clinical trial. J Clin Periodontol. 2011;38(10):925-32. http://doi.org/10.1111/j.1600-051X.2011.01760.x

16. Pradeep AR, Rao NS, Agarwal E, Bajaj P, Kumari M, Naik SB. Comparative evaluation of autologous platelet-rich fibrin and platelet-rich plasma in the treatment of 3-wall intrabony defects in chronic periodontitis: a randomized controlled clinical trial. J Periodontol. 2012;83(12):1499-507. http://doi.org/10.1902/jop.2012.110705

17. Lekovic V, Milinkovic I, Aleksic Z, Jankovic S, Stankovic P, Kenney EB et al. Platelet-rich fibrin and bovine porous bone mineral vs. platelet-rich fibrin in the treatment of intrabony periodontal defects. J Periodontal Res. 2012;47(4):409-17. http://doi.org/10.1111/j.1600-0765.2011.01446.x

18. Shah M, Patel J, Dave D, Shah S. Comparative evaluation of platelet-rich fibrin with demineralized freeze-dried bone allograft in periodontal infrabony defects: A randomized controlled clinical study. J Indian Soc Periodontol. 2015;19(1):56-60. http://doi.org/10.4103/0972-124X.145803

19. Agarwal A, Gupta ND, Jain A. Platelet rich fibrin combined with decalcified freeze-dried bone allograft for the treatment of human intrabony periodontal defects: a randomized split mouth clinical trail. Acta Odontol Scand. 2016;74(1):36-43. http://doi.org/10.3109/00016357.2015.1035672

20. Schulz KF, Altman DG, Moher D. CONSORT 2010 statement: updated guidelines for reporting parallel group randomized trials. Ann Intern Med. 2010;152(11):726-32. http://doi.org/10.7326/0003-4819-152-11-201006010-00232

21. Armitage GC. Development of a classification system for periodontal diseases and conditions. Ann Periodontol. 1999;4(1):1-6. http://doi.org/10.1902/annals.1999.4.1.1
22. Löe H. The Gingival Index, the Plaque Index and the Retention Index Systems. J Periodontol. 1967;38(6):610-6. http://doi.org/10.1902/jop.1967.38.6.610

23. Silness J, Loe H. Periodontal disease in pregnancy. II. Correlation between oral hygiene and periodontal condtion. Acta Odontol Scand. 1964;22(1):121-35. http://doi.org/10.3109/00016356408993968

24. Nibali L, Pometti D, Tu YK, Donos N. Clinical and radiographic outcomes following non-surgical therapy of periodontal infrabony defects: a retrospective study. J Clin Periodontol. 2011;38(1):50-7. http://doi.org/10.1111/j.1600-051X.2010.01648.x

25. Kim CK, Choi SH, Kim TS, Kaltschmitt J, Eickholz P. The infrabony defect and its determinants. J Periodontal Res. 2006;41(6):498-502. http://doi.org/10.1111/j.1600-0765.2006.00895.x

26. Tsitoura E, Tucker R, Suvan J, Laurell L, Cortellini P, Tonetti M. Baseline radiographic defect angle of the intrabony defect as a prognostic indicator in regenerative periodontal surgery with enamel matrix derivative. J Clin Periodontol. 2004;31(8):643-7. http://doi.org/10.1111/j.1600-051X.2004.00555.x

27. Dohan DM, Choukroun J, Diss A, Dohan SL, Dohan AJ, Mouhyi J et al. Platelet-rich fibrin (PRF): a second-generation platelet concentrate. Part I: technological concepts and evolution. Oral Surg Oral Med Oral Pathol Oral Radiol Endod. 2006;101(3):e37-44. http://doi.org/10.1016/j.tripleo.2005.07.008

28. Dohan Ehrenfest DM. How to optimize the preparation of leukocyte- and platelet-rich fibrin (L-PRF, Choukroun's technique) clots and membranes: introducing the PRF Box. Oral Surg Oral Med Oral Pathol Oral Radiol Endod. 2010;110(3):275-8. http://doi.org/10.1016/j.tripleo.2010.05.048

29. Ogihara S, Tarnow DP. Efficacy of enamel matrix derivative with freeze-dried bone allograft or demineralized freeze-dried bone allograft in intrabony defects: a randomized trial. J Periodontol. 2014;85(10):1351-60. http://doi.org/10.1902/jop.2014.130520

30. Döri F, Kovács V, Arweiler NB, Huszár T, Gera I, Nikolidakis D et al. Effect of platelet-rich plasma on the healing of intrabony defects treated with an anorganic bovine bone mineral: a pilot study. J Periodontol. 2009;80(10):1599-605. http://doi.org/10.1902/jop.2009.090058

31. Parimala M, Mehta DS. Comparative evaluation of bovine porous bone mineral. J Indian Soc Periodontol. 2010;14(2):126-31. http://doi.org/10.4103/0972-124X.70834

32. Richardson CR, Mellonig JT, Brunsvold MA, McDonnell HT, Cochran DL. Clinical evaluation of Bio-Oss: a bovine-derived xenograft for the treatment of periodontal osseous defects in humans. J Clin Periodontol. 1999;26(7):421-8. http://doi.org/10.1034/j.1600-051X.1999.260702.x 
33. Cortellini P, Tonetti MS. Clinical performance of a regenerative strategy for intrabony defects: scientific evidence and clinical experience. J Periodontol. 2005;76(3):341-50. http://doi.org/10.1902/jop.2005.76.3.341

34. Del Corso M, Sammartino G, Dohan Ehrenfest DM. Re: “Clinical evaluation of a modified coronally advanced flap alone or in combination with a platelet-rich fibrin membrane for the treatment of adjacent multiple gingival recessions: a 6-month study". J Periodontol. 2009;80(11):1694-7. http://doi.org/10.1902/jop.2009.090253

35. Liñares A, Cortellini P, Lang NP, Suvan J, Tonetti MS. Guided tissue regeneration/deproteinized bovine bone mineral or papilla preservation flaps alone for treatment of intrabony defects. II: radiographic predictors and outcomes. J Clin Periodontol. 2006;33(5):351-8. http://doi.org/10.1111/j.1600-051X.2006.00911.x

36. Ilgenli T, Dündar N, Kal BI. Demineralized freeze-dried bone allograft and platelet-rich plasma vs platelet-rich plasma alone in infrabony defects: a clinical and radiographic evaluation. Clin Oral Investig. 2007;11(1):51-9. http://doi.org/10.1007/s00784-006-0083-y

37. Klein F, Kim TS, Hassfeld S, Staehle HJ, Reitmeir P, Holle R et al. Radiographic defect depth and width for prognosis and description of periodontal healing of infrabony defects. J Periodontol. 2001;72(12):1639-46. http://doi.org/10.1902/jop.2001.72.12.1639

38. Sharma A, Pradeep AR. Treatment of 3-wall intrabony defects in patients with chronic periodontitis with autologous platelet-rich fibrin: a randomized controlled clinical trial. J Periodontol. 2011;82(12):1705-12. http://doi.org/10.1902/jop.2011.110075

39. Sculean A, Chiantella GC, Windisch P, Gera I, Reich E. Clinical evaluation of an enamel matrix protein derivative (Emdogain) combined with a bovine-derived xenograft (Bio-Oss) for the treatment of intrabony periodontal defects in humans. Int J Periodontics Restorative Dent. 2002;22(3):259-67. 